\title{
ANTENNA RESISTANCE
}

By L. W. Austin

A preliminary note on this subject ${ }^{1}$ showed that, in accordance with the Hertzian theory, the antenna resistance varies approximately inversely as the square of the wave length up to a point which is slightly less than twice the fundamental wave length of the antenna. Beyond this point the resistance again rises nearly in direct proportion to the wave length. This increase in resistance with wave length is not explained by the theory and is not accompanied by any increase in radiation. It is therefore supposed to be connected in some way with the losses due to the currents in the earth beneath and in the neighborhood of the antenna, although this view-that the earth current resistance increases with wave length-is not supported by independent investigations of earth current resistance. $^{2}$

The peculiar nature of the results obtained in the original investigation, using an artificial antenna, has made it desirable to verify the results by other methods. The circuits employed in the first investigation are shown in Fig. I. The method, in brief, is the substitution of an air condenser in place of the antenna and ground, keeping the inductance common to both circuits and introducing resistance in the condenser circuit until the current becomes the same as that observed when the antenna and ground were used. In the figure $A$ is the antenna, $E$ the ground, $L_{1}$ the tuning inductance, $C_{1}$ an air condenser for tuning to very short wave lengths, $T h$ a thermoelement, $G$ a galvanometer, and $C_{2}$ a variable air condenser set at the capacity of the antenna to be measured. A

${ }^{1} \mathrm{~J}$. of Washington Academy, r, p. 9; $191 \mathrm{r}$.

${ }^{2} \mathrm{H}$. True: Jahrbuch d. drahtlosen Telegraphie, 5, p. 125; 1912. 
small inductance $L_{2}$ was sometimes inserted in circuit with the condenser $C_{2}$ to represent the antenna inductance. This has little influence on the results and at least for the longer wave lengths may be perfectly well omitted. $S_{1}$ and $S_{2}$ are switches for connecting either the antenna and ground or $C_{2}$ to the rest of the circuit. $R$ is a resistance introduced in the circuit $C_{2}$ to bring down the thermoelement deflection to the same value as that observed when the antenna and ground are in circuit. The high frequency resistance consists of separate units of fine constantan wire inserted in

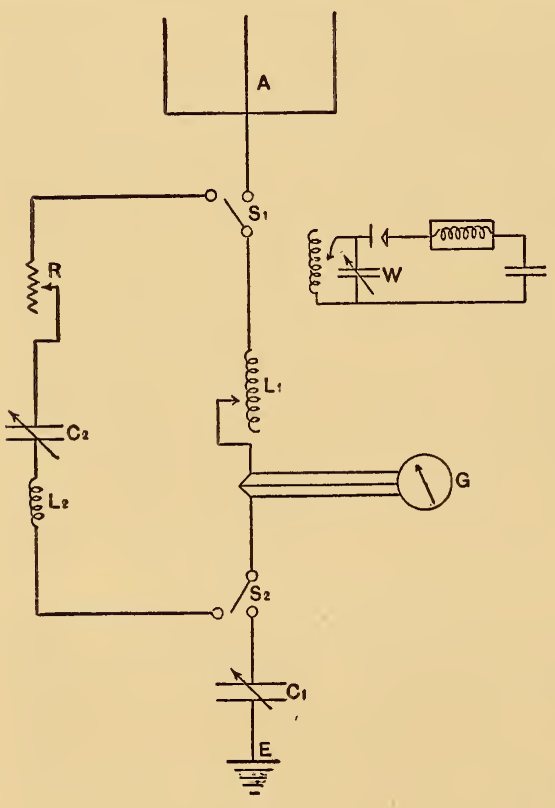

Fig. 1 mercury cups. The measurement circuit is excited by a buzzer-driven wave meter $W$ of the ordinary type.

The second method employed was the well-known decrement method of determining resistance. Here the antenna was excited by a wave meter of known logarithmic decrement provided with a buzzer. The reading of the condenser of the wave meter was noted for maximum deflection of the thermoelement in the antenna and also when the wave meter was thrown out of resonance by an amount sufficient to reduce the antenna thermoelement reading to onehalf. The expression for the sum of the decrements is, then,

$$
\delta_{1}+\delta_{2}=\pi \frac{C_{m}-C}{C_{m}}
$$

where $C_{m}$ denotes the condenser reading for maximum deflection and $C$ the reading for which the deflection is reduced to one-half. As $\delta_{1}$, the wave meter decrement, is known, the equation serves to give the value of the antenna decrement $\delta_{2}$. The resistance of the antenna is obtained from the decrement according to the equation

$$
\dot{R}=2 n L \delta_{2} \text {. }
$$


A third method is based on the Bjerknes equation of the current square in the second of two coupled circuits.

I)

$$
I^{2}=\frac{\text { Constant }}{\delta_{1}{ }^{2} \delta_{2}+\delta_{2}{ }^{2} \delta_{1}} .
$$

Here $I$ represents the R. M. S. current in a circuit excited inductively by another, $\delta_{1}$ the decrement of the first circuit, and $\delta_{2}$ that of the second. If the decrement of the first circuit is very great compared to that of the second, the second term of the denominator may be neglected, and

$$
I^{2}=\frac{\text { Constant }}{\delta_{1}^{2} \delta_{2}} ;
$$

that is, doubling the resistance in the second circuit will reduce the current square to one-half. If the antenna is excited by a highly damped circuit, the readings of a galvanometer attached to a thermoelement in the antenna will be reduced to one-half when a resistance equal to the total antenna resistance is inserted in the antenna. In practice it is impossible to obtain an exciting circuit whose decrement is great enough to allow using this method in connection with an antenna unless a correction be made for the second term of the denominator of equation $\mathrm{I}$.

\section{TABLE I}

Correction to be Added to Observed Resistance Required to Reduce $I^{2}$ to One-half for Various Ratios of Decrement in Half Deflection Method

\begin{tabular}{c|c}
\hline$\frac{\delta_{1}}{\grave{o}_{2}}$ & Correction (per cent) \\
\hline 3 & 42 \\
4 & 38 \\
5 & 28 \\
7 & 23.5 \\
10 & 17.5 \\
15 & 12 \\
20 & 8.7 \\
30 & 6.2 \\
50 & 4.2 \\
\hline
\end{tabular}


Corrections for various ratios of $\delta_{1}$ to $\delta_{2}$ are given in Table I. These corrections are to be added to the resistance which reduces the value of $I^{2}$ to one-half to obtain the actual antenna resistance. For rough testing in the case of quenched gap sets this correction may be taken as about 20 per cent. This assumes four waves in the primary train.

It is to be observed that in the last two methods the resistances of the inductance of the antenna and of the inserted thermoelement are added to the resistance of the antenna and ground alone as measured by the artificial antenna method. The high

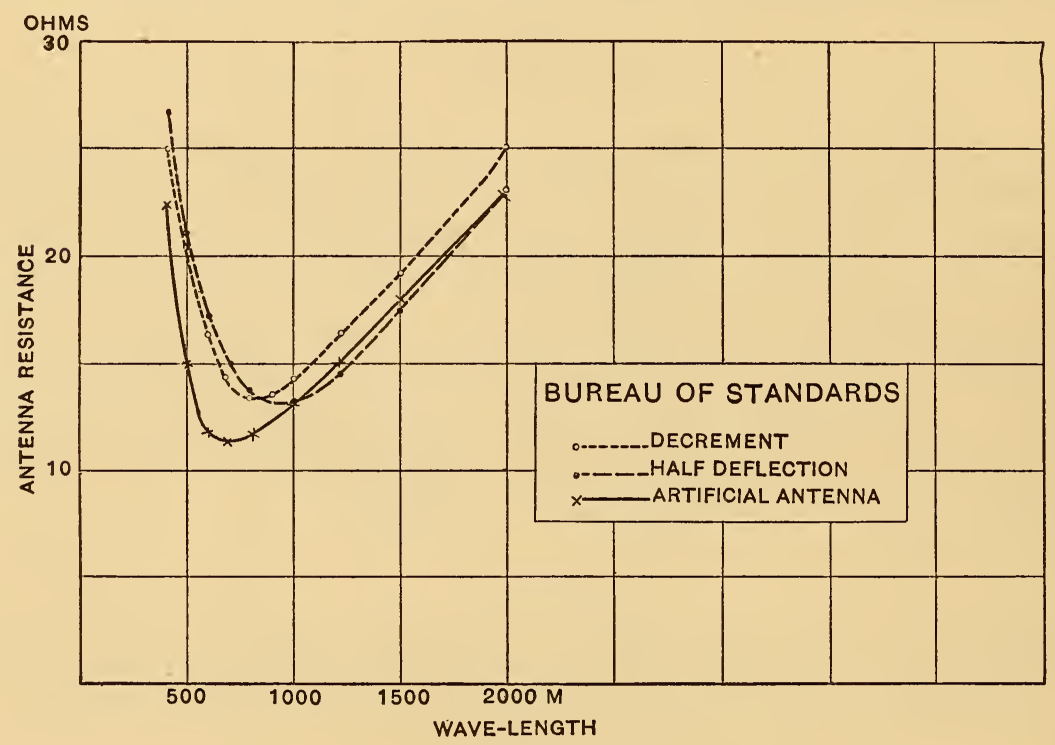

Fig. 2

frequency resistance of the inductance at short wave lengths may amount to considerable, therefore it is to be expected that these methods will give higher values than the one first used.

Measurements by these three methods have been made on the antenna at the Bureau of Standards and the results are shown in Fig. 2. As was to be expected from what has been already said, the artificial antenna method gives in general the lowest values, while the decrement method gives a curve lying above the artificial antenna curve showing a difference of a little more than I ohm at the longer wave lengths, and for the shorter wave 
lengths a still greater difference on account of the increase in the resistance of the antenna inductance with decreasing wave length. The half deflection method gives a curve which follows the decrement curve closely at the shorter wave lengths and the artificial antenna curve at the longer wave lengths. This is undoubtedly due to errors in the determination of the ratio of the decrements in the quenched gap and antenna circuits. These errors are not at all surprising when it is remembered that the decrement of a spark circuit has been shown by experiment to be not by any means logarithmic in its character. ${ }^{1}$

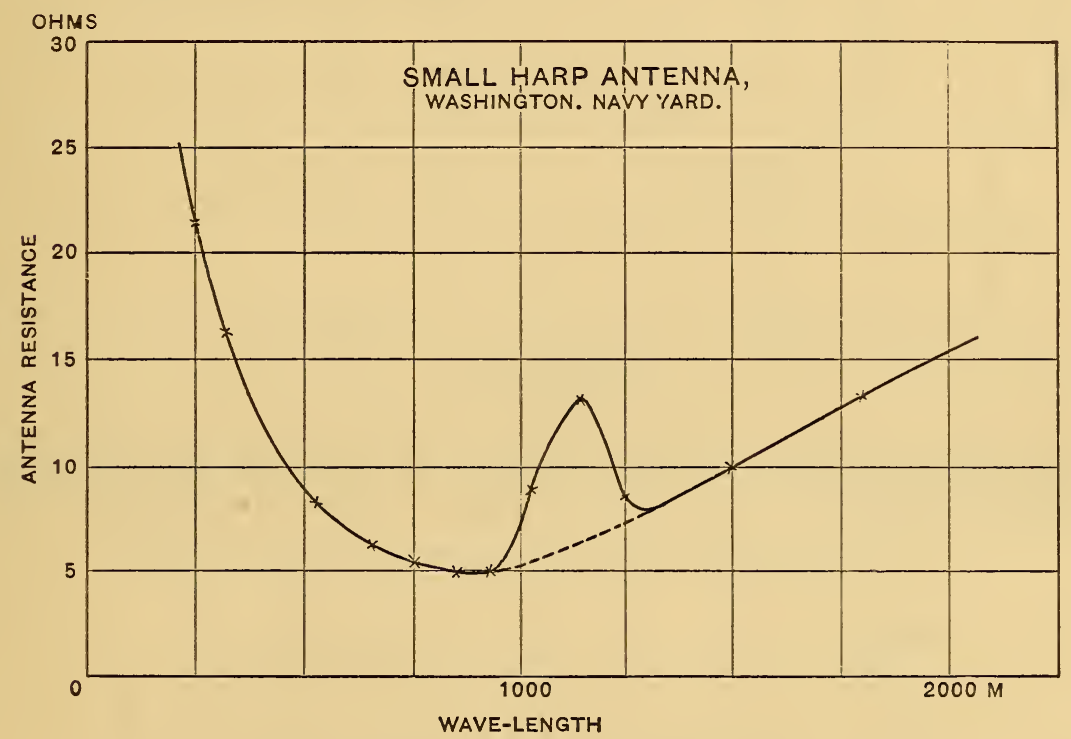

Fig. 3

Of the three methods, that of the artificial antenna is undoubtedly the most accurate, while that of the half deflection is the least so. The latter, however, is by far the simplest for ordinary station testing, as it may be applied without the use of any extra apparatus, the readings being taken with the regular hot wire meter, sufficient suitable high frequency resistance being added to reduce the square of the antenna meter current to one-half. ${ }^{2}$

\footnotetext{
1 J. Zenneck: Ann. der Phys., 13, p. 822; I904.

${ }^{2}$ For wave lengths above 500 meters it may be assumed without great error that the high frequency resistance of No. 20 ( $0.8 \mathrm{I}$ millimeters) Advance or manganin wire is the same as its direct-current resistance. The resistance must of course be made up noninductively and preferably in a number of separate tunits. This wire will carry 7 to 8 amperes without heating.
} 
Since the preliminary paper was published, the resistances of a large number of antennas, both at shore stations and on ships of

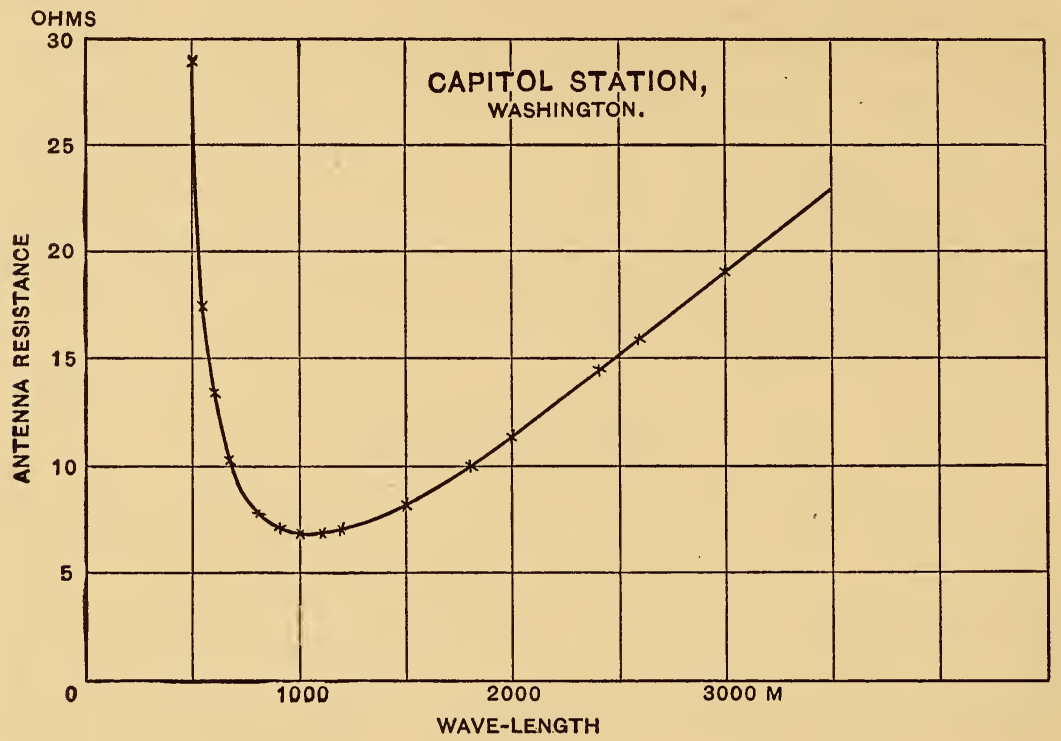

Fig. 4

the United States Navy, have been measured by the artificial antenna method. The results of some of these are shown by the

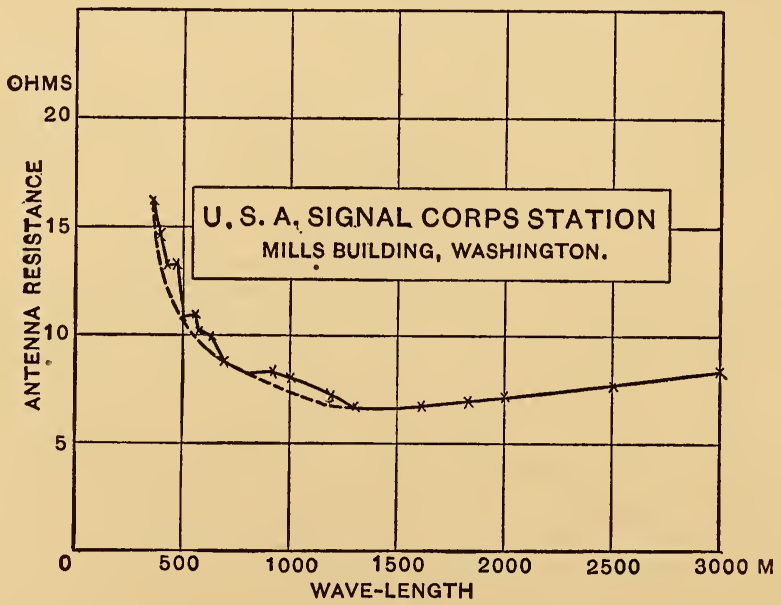

Fig. 5

curves of Figs. 3 to 7 . The curves in general are self-explanatory, indicating that the minimum resistance in a well-installed 
ship set should not rise much above 2 ohms. It appears to be difficult, if not impossible, to bring the resistance of land stations as low as this. The irregularities in the curve of the Signal Corps antenna installed above the roof of the Mills Building in

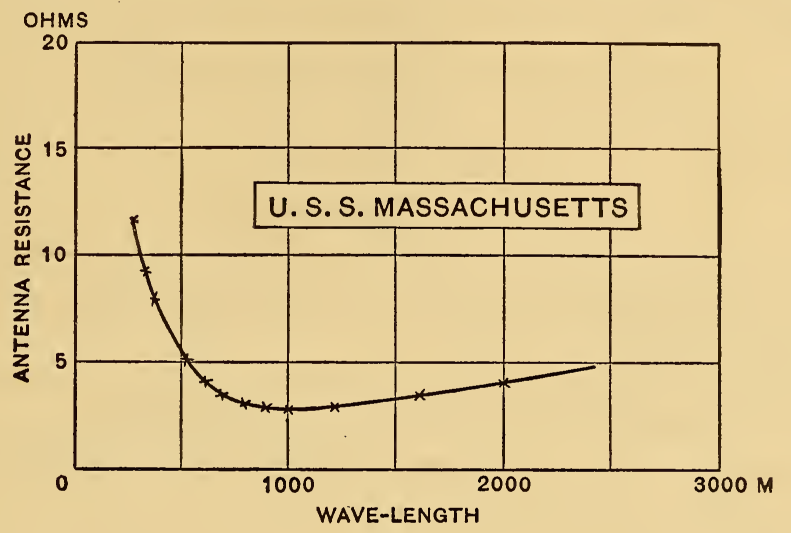

Fig. 6

Washington show clearly the effects of resonance in the metal portions of the building, while the curve for the harp antenna at the Washington Navy Yard shows a large increase in resistance at the point where it is in resonance with the main antenna of

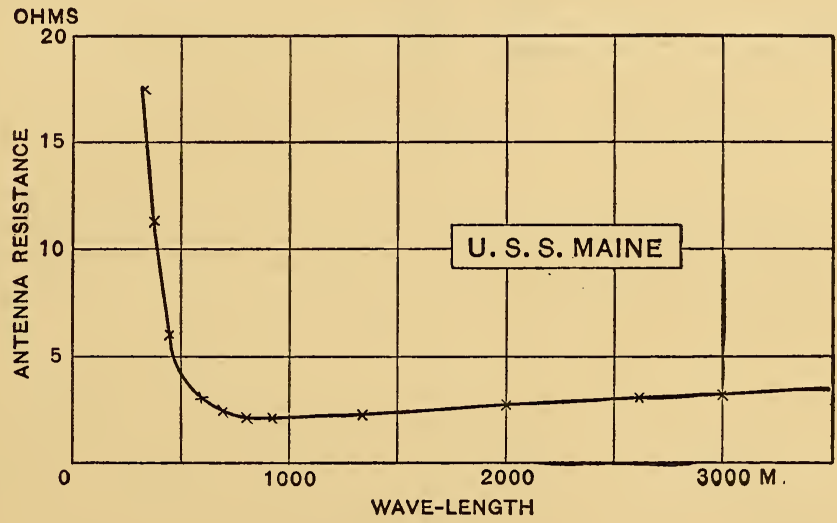

Fig. 7

the wireless station. These two examples prove the necessity of taking care that no wave length used shall correspond to the period of any metallic structure in the neighborhood of the antenna, such as the steel masts and rigging of ships. 
TABLE II

\begin{tabular}{|c|c|c|c|c|}
\hline Stations & Antenna type & $\begin{array}{c}\text { Length } \\
\text { (meters) }\end{array}$ & $\underset{\mu \mathrm{f}}{\text { Capacity }}$ & $\underset{\text { (meters) }}{\text { Height }}$ \\
\hline Bureau of Standards. . & Harp.... & 42.6 & 0.00126 & 54.8 \\
\hline Signal Corps..................... & Flat top...... & 44.5 & .00171 & 39.6 \\
\hline 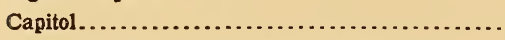 & .....do.......... & 85 & .00232 & 44.2 \\
\hline Washington Navy Yard (small antenna).. & Harp............ & 51.8 & .00073 & 54.8 \\
\hline U. S. S. Maine............................. & Flat top..... & 32.9 & .00125 & 38.4 \\
\hline U. S. S. Massachusetts.......... & .....do......... & 24.4 & .00110 & 39 \\
\hline
\end{tabular}

The observations for this paper have been taken for the most part by my assistants, Mineratti and Scanlon, chief electricians, United States Navy.

U. S. NAVAL Radiotelegraphic Laboratory, WASHINGTON, March I, I9I2. 